\title{
Optimal Construction of Edge-Disjoint Paths in Random Regular Graphs
}

\author{
Alan M. Frieze* Lei Zhao ${ }^{\dagger}$
}

\begin{abstract}
Given a graph $G=(V, E)$ and a set of $\kappa$ pairs of vertices in $V$, we are interested in finding for each pair $\left(a_{i}, b_{i}\right)$, a path connecting $a_{i}$ to $b_{i}$, such that the set of $\kappa$ paths so found is edge-disjoint. (For arbitrary graphs the problem is $\mathcal{N} \mathcal{P}$-complete, although it is in $\mathcal{P}$ if $\kappa$ is fixed.)

We present a polynomial time randomized algorithm for finding the optimal number of edge disjoint paths (up to constant factors) in the random regular graph $G_{n, r}$, for $r$ sufficiently large. (The graph is chosen first, then an adversary chooses the pairs of endpoints.)
\end{abstract}

\section{Introduction}

Given a graph $G=(V, E)$ with $n$ vertices, and a set of $\kappa$ pairs of vertices in $V$, we are interested in finding for each pair $\left(a_{i}, b_{i}\right)$, a path connecting $a_{i}$ to $b_{i}$, such that the set of $\kappa$ paths so found is edge-disjoint.

For arbitrary graphs the related decision problem is $\mathcal{N} \mathcal{P}$-complete, although it is in $\mathcal{P}$ if $\kappa$ is fixed - Robertson and Seymour [23]. Nevertheless, this negative result can be circumvented for certain classes of graphs. Peleg and Upfal [22] presented a polynomial time algorithm for the case where $G$ is a (sufficiently strong) bounded degree expander graph, and $\kappa \leq n^{\epsilon}$ for a small constant $\epsilon$ that depends on the expansion property of the graph. (A precise upper bound for $\epsilon$ was not computed, but it is clearly less that $1 / 3$ ). This result has been improved and extended by Broder, Frieze, and Upfal [10,11], Frieze [13], Leighton and Rao [18] and Leighton, Rao and Srinivasan [19, 20]: $G$ still has to be a (sufficiently strong) bounded degree expander but $\kappa$ can now grow as fast as $n /(\ln n)^{\theta}$, where $\theta$ depends only on the expansion properties of the input graph, but is at least 2 .

In random graphs Shamir and Upfal have shown in [24] that any set of up to $O(\sqrt{n})$ pairs can be connected via vertex-disjoint paths; similar results using efficient flow techniques were also obtained by Hochbaum [15]. These results were proved for graphs with $m \geq K n \log n$ random edges, where $K$ is a sufficiently large constant. In these two papers the pairs are chosen first and then the graph is randomly generated. Thus they do not deal with the problem of satisfying all sets of $\kappa$ pairs.

Let $D$ be the median distance between pairs of vertices in $G$. Clearly it is not possible to connect more than $O(m / D)$ pairs of vertices by edge-disjoint paths, for all choices of pairs,

\footnotetext{
*Department of Mathematical Sciences, Carnegie-Mellon University, Pittsburgh, PA 15213. Supported in part by NSF grant CCR9530974. E-mail: alan@random.math. cmu.edu.

${ }^{\dagger}$ Department of Mathematical Sciences, Carnegie-Mellon University, Pittsburgh, PA 15213. Supported in part by NSF grant CCR9530974. E-mail: lzhao@andrew.cmu.edu
} 
since some choice would require more edges than all the edges available. In the case of bounded degree expanders, this absolute upper bound on $\kappa$ is $O(n / \log n)$. The results mentioned so far use only a vanishing fraction of the set of edges of the graph, thus are far from reaching this upper bound. Broder, Frieze, Suen and Upfal [8] have proved that for the model of random graphs, $G_{n, m}$, the absolute upper bound is achievable to within a constant factor, but only if the average degree is at least $\ln n$. In this work, we show that this result holds when the minimum degree is a large enough constant. Without significant loss of generality, we consider random regular graphs and present an algorithm that constructs the required paths in polynomial time.

As usual, let $G_{n, p}$ denote a random graph with vertex set $\{1,2, \ldots, n\}=[n]$ in which each possible edge is included independently with probability $p$, and let $G_{n, m}$ denote a random graph also with vertex set $[n]$ and exactly $m$ edges, all sets of $m$ edges having equal probability. Let $G_{n, r}$ denote a random graph again with vertex set $[n]$ which is chosen uniformly at random from all possible $r$-regular graphs.

Our main result is formulated in the following theorem.

Theorem 1 Let $r$ be a sufficiently large constant. Then, as $n \longrightarrow \infty$, the graph $G_{n, r}$ has the following property whp: there exist positive absolute constants $\alpha, \beta$ such that for all sets of pairs of vertices $\left\{\left(a_{i}, b_{i}\right) \mid i=1, \ldots, \kappa\right\}$ satisfying:

(i) $\kappa=\left\lceil\alpha r n / \log _{r} n\right\rceil$,

(ii) for each vertex $v,\left|\left\{i: a_{i}=v\right\}\right|+\left|\left\{i: b_{i}=v\right\}\right| \leq \beta r$,

there exist edge-disjoint paths in $G$, joining $a_{i}$ to $b_{i}$, for each $i=1,2, \ldots, \kappa$. Furthermore, there is an $O\left(n^{3}\right)$ time randomized algorithm for constructing these paths.

This result is the best possible up to constant factors. The median distance between pairs of vertices in $G$ is $\Omega\left(\log _{r} n\right)$. The need for $\beta<1$ is discussed in [8].

The analogous problem of finding vertex disjoint paths in random graphs is dealt with in $[9]$.

\section{Preliminaries}

The paper contains a few unspecified absolute constants of which $\alpha$ above is the first. Exact values could be given but it is easier for us and the reader if we simply give the relations between them. New constants will be introduced as $C_{0}, \ldots$ without further comment. Furthermore, specific constants have been chosen for convenience. We made no attempt to optimize them, and, in general, we only claim that inequalities dependent on $n$ or $r$ hold for $n$ or $r$ sufficiently large.

For a graph $G=(V, E)$ and $v \in V$ we let $d_{G}(v)$ denote the degree of $v$ in $G$. We use $\delta(G)$ and $\Delta(G)$ to denote the smallest and largest degrees respectively. For a set $S \subseteq V$ we let $\bar{S}=V \backslash S$ and define its neighbor set, $N_{G}(S)$, as

$$
N_{G}(S)=\{v \in \bar{S}: \exists w \in S \text { such that }\{v, w\} \in E\} .
$$

For $S, T \subseteq V$ we let $e_{G}(S, T)$ denote the number of edges with one end in $S$ and one end in $T$. For $S \subseteq V$, we use $G[S]$ to denote the subgraph of $G$ induced by $S$. 
The Chernoff bounds on the tails of the $\operatorname{Binomial~} \operatorname{Bin}(n, \theta)$ that we use are

$$
\begin{aligned}
& \operatorname{Pr}(\operatorname{Bin}(n, \theta) \leq(1-\epsilon) n \theta) \leq e^{-\epsilon^{2} n \theta / 2}, \\
& \operatorname{Pr}(\operatorname{Bin}(n, \theta) \geq(1+\epsilon) n \theta) \leq e^{-\epsilon^{2} n \theta / 3},
\end{aligned}
$$

valid for $0 \leq \epsilon \leq 1$.

\section{Overview of the algorithm}

Our algorithm divides naturally into the four phases sketched below.

Phase 0: Partition $G$ into six edge-disjoint graphs $G_{i}=\left(V_{i}, E_{i}\right), 1 \leq i \leq 6$. Phase 1 will use only the graph $G_{1}$; Phase 2 will use only the graphs $G_{2}, G_{3}, G_{4}$ and $G_{5}$; and Phase 3 will use only the graph $G_{6}$. The partition is such that $V_{1}=V$ but $V_{2}=\cdots=V_{6} \subseteq V$ with $\left|V_{2}\right| \geq n-\epsilon n$, where $\epsilon=\epsilon(r)$ is a small constant.

Phase 1: Choose a random set $Z=\left\{z_{1}, \ldots, z_{2 \kappa}\right\}$ of $2 \kappa$ points in $V_{2}$. Connect the endpoints $\left\{\left(a_{i}, b_{i}\right) \mid i=1, \ldots, \kappa\right\}$ to the newly chosen points in an arbitrary manner via edge-disjoint paths in $G_{1}$ using a flow algorithm. Let $\tilde{a}_{i}$ (resp. $\tilde{b}_{i}$ ) be the vertex connected to $a_{i}$ (resp. $b_{i}$ ). The original problem is now reduced to finding edge-disjoint paths from $\tilde{a}_{i}$ to $\tilde{b}_{i}$ for each $i$. (This randomization was used in [10] and has its roots in Valiant's routing algorithm [26].)

Phase 2: We split this into parts (a),(b).

(a) For each $z_{i}, 1 \leq i \leq 2 \kappa$, we do a random walk of length

$$
\tau_{0}=\left\lceil 10 \log _{r} n\right\rceil
$$

starting at $z_{i}$ in $G_{2}$. The terminating endpoint of this walk will be denoted by $\hat{a}_{j}$ if $z_{i}=\tilde{a}_{j}$ and by $\hat{b}_{j}$ if $z_{i}=\tilde{b}_{j}$. Ater we complete a walk we remove the edges from the corresponding graph.

(b) For each $i$ in turn, we first do a random walk starting at $\hat{b}_{i}$ and terminating at $b_{i}^{*}$ in $G_{4}$. Then we repeatedly do a certain type of random walk in $G_{3}$ starting from $\hat{a}_{i}$ until one of these walks ends at $b_{i}^{*}$. We keep the last walk as our path from $\hat{a}_{i}$ to $b_{i}^{*}$ and remove all edges seen in these walks from $G_{3}$ and $G_{4}$ respectively.

Most pairs $\left(\tilde{a}_{i}, \tilde{b}_{i}\right)$ will be successfully connected in this phase. For such a pair, the final path from $a_{i}$ to $b_{i}$ is the concatenation of the paths (after removing cycles if necessary.) from $a_{i}$ to $\tilde{a}_{i}$ and from $b_{i}$ to $\tilde{b}_{i}$ found in Phase 1 , the paths from $\tilde{a}_{i}$ to $\hat{a}_{i}$, from $\hat{a}_{i}$ to $b_{i}^{*}$, from $b_{i}^{*}$ to $\hat{b}_{i}$ and from $\tilde{b}_{i}$ to $\hat{b}_{i}$ which are found in this stage. It is important in our analysis to ensure that random walks are done on graphs of high minimum degree. We use $G_{5}$ as a backup for ensuring that this is done.

Phase 3: At the end of Phase 2, whp, there will be at most $n /(\ln n)^{C_{0}}$ pairs $\left(\tilde{a}_{i}, \tilde{b}_{i}\right)$ which have not been joined by paths. We use the algorithm of [10] to join them by edge disjoint paths, using only the edges of $G_{6}$, and then construct the final paths from $a_{i}$ to $b_{i}$ as above.

To prove Theorem 1 it suffices to show that for almost every $G_{n, r}$ :

- Phases 0 and 1 will succeed for all choices of $a_{1}, \ldots, b_{\kappa}$ and almost every choice of $z_{1}, \ldots, z_{2 \kappa}$.

- Phases 2 and 3 are successful for almost every choice of $z_{1}, \ldots, z_{2 \kappa}$ and any bijection $\left\{\tilde{a}_{1}, \ldots, \tilde{a}_{\kappa}, \tilde{b}_{1}, \ldots, \tilde{b}_{k}\right\} \longrightarrow\left\{z_{1}, \ldots, z_{2 \kappa}\right\}$ 
Note that to prove these facts we have to consider only one experiment, namely choose $G_{n, r}$ at random, choose $a_{1}, \ldots, b_{\kappa}$ arbitrarily and then $z_{1}, \ldots, z_{2 \kappa}$ at random. From this we can deduce that almost every $G_{n, r}$ is such that for all choices of $a_{1}, \ldots, b_{\kappa}$ and almost every choice of $z_{1}, \ldots, z_{2 \kappa}$, we can find edge-disjoint paths $a_{i}-\tilde{a}_{i}-\hat{a}_{i}-b_{i}^{*}-\hat{b}_{i}-\tilde{b}_{i}-b_{i}$ for $1 \leq i \leq \kappa$.

\section{Detailed description of the algorithm}

The input to our algorithm is a random graph $G_{n, r}$ and a set of pairs of vertices $\left\{\left(a_{i}, b_{i}\right) \mid i=\right.$ $1, \ldots, \kappa\}$ satisfying the premises of Theorem 1 . The output is a set of $\kappa$ edge-disjoint paths, $P_{1}, \ldots, P_{\kappa}$ such that $P_{i}$ connects $a_{i}$ to $b_{i}$.

\subsection{Phase 0.}

We start by partitioning $G$ into six edge-disjoint graphs $G_{i}=\left(V_{i}, E_{i}\right)$, for $1 \leq i \leq 6$. Phase 1 will use only $G_{1}$; Phase 2 will use only $G_{2}, G_{3}, G_{4}$ and $G_{5}$; Phase 3 will use only $G_{6}$. The partition is such that $V_{1}=V$ but $V_{2}=\cdots=V_{6} \subseteq V$ with $\left|V_{2}\right| \geq n-\epsilon n$, where $\epsilon=\epsilon(r)$ is a small constant.

In this construction, we use the notion of a $k$-core. The $k$-core of a collection of graphs $H_{1}, H_{2}, \ldots, H_{l}$ on the same vertex set $V$ is the largest subset of $V$ which induces a subgraph of minimum degree at least $k$ in each of $H_{1}, H_{2}, \ldots, H_{l}$. It is unique and can be found by repeatedly removing vertices which have degree less than $k$ in one of the $l$ induced graphs. This continues until there are no vertices left or minimum degree $k$ is achieved.

The algorithm below starts by constructing preliminary versions of these graphs, denoted $G_{i}^{\prime}$ for $1 \leq i \leq 6$. Then edges and vertices are deleted from $G_{2}^{\prime}, \ldots, G_{6}^{\prime}$ in order to achieve certain minimum degree properties.

\section{Algorithm SPLIT}

2. begin

3. Divide $E$ into $E_{i}^{\prime}, 1 \leq i \leq 6$ by placing each edge of $E$ independently with probability $1 / 6$ into each of $E_{i}^{\prime}$ for $1 \leq i \leq 6$.

4. $\quad$ for $1 \leq i \leq 6$ set $G_{i}^{\prime} \leftarrow\left(V, E_{i}^{\prime}\right)$

5. $\quad K \leftarrow\lceil r / 7\rceil-$ core of $G_{1}^{\prime}$

6. $\quad$ for $2 \leq i \leq 6 \operatorname{set} G_{i} \leftarrow G_{i}^{\prime}[K]$

7. $\quad K \leftarrow\lceil r / 7\rceil-$ core of $G_{2}, \ldots, G_{6}$.

8. $\quad$ for $2 \leq i \leq 6 G_{i}=\left(V_{i}, E_{i}\right) \leftarrow G_{i}[K]$.

9. end SPLIT

We will show later (Lemma 4) that whp this algorithm terminates with $|K| \geq n-\epsilon n$ where $\epsilon$ is small. Note that SPLIT ensures that

- The final graphs $G_{i}, i=2, \ldots, 6$ have the same vertex set $K$.

- Every $v \in K$ has degree at least $\lceil r / 7\rceil$ in each $G_{i}, 1 \leq i \leq 6$.

- Every $v \in K$ has degree at most $2 r / 7$ in each $G_{i}, 2 \leq i \leq 6$.

- If $v \in V \backslash K$ then $d_{G_{1}}(v)=d_{G}(v)=r$. 


\subsection{Phase 1.}

Choose $z_{1}, z_{2}, \ldots, z_{2 \kappa} \in V_{2}$ uniformly and randomly without replacement. Let $Z$ denote the set $\left\{z_{1}, z_{2}, \ldots, z_{2 \kappa}\right\}$. We are going to replace the problem of finding paths from $a_{i}$ to $b_{i}$ by that of finding paths from $\tilde{a}_{i}$ to $\tilde{b}_{i}$, where $\left\{\tilde{a}_{1}, \tilde{b}_{1}, \tilde{a}_{2}, \tilde{b}_{2}, \ldots, \tilde{a}_{\kappa}, \tilde{b}_{\kappa}\right\}=Z$. Let $A$ denote the set $\left\{a_{1}, b_{1}, a_{2}, b_{2}, \ldots, a_{\kappa}, b_{\kappa}\right\}$.

We connect $A$ to $Z$ via edge-disjoint paths in the graph $G_{1}$ using network flow techniques. We construct a network as follows

- Each undirected edge of $G_{1}$ gets capacity 1.

- Each $v \in V$ becomes a source of capacity $\mid\left\{i: a_{i}=v\right.$ or $\left.b_{i}=v\right\} \mid$ and each member of $Z$ becomes a sink of capacity 1 .

Then we find a flow from $A$ to $Z$ that satisfies all demands. Since the maximum flow has integer values, it decomposes naturally into $|A|$ edge-disjoint paths (together perhaps with some cycles). If a path joins $a_{i}$ to $z \in Z$, then we let $\tilde{a}_{i}=z$. Similarly, if a path joins $b_{i}$ to $z \in Z$, then we let $\tilde{b}_{i}=z$.

Thus Phase 1 finds edge-disjoint paths $W_{i}^{(1)}$ from $a_{i}$ to $\tilde{a}_{i}$ and $W_{i}^{(6)}$ from $\tilde{b}_{i}$ to $b_{i}, 1 \leq$ $i \leq \kappa$, where the vertices $\tilde{a}_{1}, \tilde{b}_{1}, \tilde{a}_{2}, \tilde{b}_{2}, \ldots, \tilde{a}_{\kappa}, \tilde{b}_{\kappa} \in V_{2}$ are chosen uniformly at random without replacement. On the other hand there may be some difficult conditioning involved in the pairing of $\tilde{a}_{i}$ with $\tilde{b}_{i}, 1 \leq i \leq \kappa$. We deal with this in Phase 2(a).

\subsection{Phase 2.}

\subsubsection{Algorithm GenPaths.}

We construct edge-disjoint paths connecting $\tilde{a}_{i}, \tilde{b}_{i}$ for $1 \leq i \leq \kappa$ in this phase using random walks. A random walk on an undirected graph (or multigraph) $G=(V, E)$ is a Markov chain $\left\{X_{t}\right\}$ on $V$ associated with a particle that moves from vertex to vertex according to the following rule: The probability of a transition from vertex $v$, of degree $d_{v}$ to a vertex $w$ is $1 / d_{v}$ if $\{v, w\} \in E$ and 0 otherwise. (For multigraphs, each edge out of a vertex is an equally likely exit; loops are counted as two exits.) Its stationary distribution, denoted by $\pi$ or $\pi(G)$, is given by

$$
\pi_{v}=\frac{d_{v}}{2|E|}
$$

A trajectory $W$ of length $\tau$ is a sequence of vertices $\left[w_{0}, w_{1}, \ldots, w_{\tau}\right]$ such that $\left\{w_{t}, w_{t+1}\right\} \in E$ for $1 \leq t<\tau$. The Markov chain induces a probability distribution on trajectories in the usual way. We use $\operatorname{Pr}_{G}^{(\tau)}(a, b)$ to denote the probability that a random walk in $G$ of length $\tau$ starting at $a$ terminates at $b$.

There is some possible conditioning involved in the pairing of $\tilde{a}_{i}$ with $\tilde{b}_{i}$. To break this we first do random walks $W_{i}^{(2)}, W_{i}^{(5)}$ starting from $\tilde{a}_{i}, \tilde{b}_{i}, 1 \leq i \leq \kappa$ in graph $\Gamma_{2}$. Here $\Gamma_{j}=$ $\left(K_{j}, E_{j}\right), j=2, \ldots, 5$ denotes $G_{j}$ after the deletion of some vertices and edges. We do these in the order $z_{1}, z_{2}, \ldots, z_{2 \kappa}$. The walks are sufficiently long that their endpoints $\hat{a}_{i}, \hat{b}_{i}$ are essentially independent of $\tilde{a}_{i}$ and $\tilde{b}_{i}$. The edges of each such a walk are deleted before the next walk starts. This keeps the walks edge-disjoint. This constitutes Phase 2(a).

Then for $i=1, \ldots, \kappa$ we do the following: We construct a random walk $W_{i}^{(4)}$ starting at $\hat{b}_{i}$ in $\Gamma_{4}$ of length $\tau_{0}$, the endpoint is denoted by $b_{i}^{*}$. (This might seem unnecessary and we explain why we do it later.) Then in Step 20, we execute the subroutine WALK to generate a 
set of random walks $\hat{W}_{1}, \ldots, \hat{W}_{l}$ starting at $\hat{a}_{i}$ in $\Gamma_{3}$. The last walk $\hat{W}_{l}$ will end at $b_{i}^{*}$ and be accepted as $W_{i}^{(3)}$. Finally, we delete $\hat{W}_{1}, \ldots, \hat{W}_{l}$ from $G_{3}$ and delete $W_{i}^{(4)}$ from $G_{2}$.

It is important for the above analysis to ensure that our random walks take place on graphs with sufficiently high minimum degree. The obvious reason for this is that we do not wish to isolate any vertex that needs to be an endpoint of a walk. We also require that the walks take place on graphs which are strong expanders. For us, this requires a high minimum degree. In general we ensure that graphs supporting the random walks have minimum degree at least $r / 10$. We manage this by the simple artifact of removing vertices of low degree until the graph has none left. (We could empty a graph this way, but we show later that this does not often happen.)

If the proposed start vertex $v$ of a walk is of low degree then we try to connect it back to vertices of large degree (those in $K_{j}$ ) by a path in $G_{5}$. The terminal endpoint of this walk is denoted by $v^{\prime}$. (Note that this paths is not of length $\tau_{0}$, we expect it to be much shorter). We use a subroutine CONNECTBACK for this purpose. We do not expect to succeed all the time and our failures are kept in a set $L$ for later consideration.

The walk from $a_{i}$ to $b_{i}$ is then the catenation of walks $W_{i}^{(t)}, t=1, \ldots, 6$. These walks may each include a short walk $W_{C B}$ at the beginning provided by CONNECTBACK.

For $z \in Z$ we use the notation $\phi(z)=i$ to indicate $z \in\left\{\tilde{a}_{i}, \tilde{b}_{i}\right\}$. 


\section{Algorithm GenPaths}

2. begin

3. $\quad$ for $i=1$ to $2 \kappa$ do

4. $\quad K_{2} \leftarrow\lceil r / 10\rceil$-core of $G_{2}, \Gamma_{2} \leftarrow G_{2}\left[K_{2}\right]$.

5. $\quad$ if $z_{i} \notin K_{2}$ then

6. $\quad$ Execute ConnectBack $\left(K_{2}, z_{i}, z_{i}^{\prime}, \phi\left(z_{i}\right), W_{C B}\right) \mathbf{f i}$

7. $\quad$ if $j=\phi\left(z_{i}\right) \notin L$ then

8. $\quad$ Let $\theta=2$ if $z_{i}=\tilde{a}_{j}$ and $\theta=5$ if $z_{i}=\tilde{b}_{j}$. Construct random walk $W_{j}^{(\theta)}$ starting at $z_{i}^{\prime}$ in $\Gamma_{2}$. The terminal endpoint is $\hat{a}_{j}$ if $z_{i}=\tilde{a}_{j}$ and $\hat{b}_{j}$ otherwise.

9.

10. $W_{j}^{(\theta)} \leftarrow\left(W_{C B}, W_{j}^{(\theta)}\right), G_{2} \leftarrow G_{2} \backslash E\left(W_{j}^{(\theta)}\right)$

11. od

12. for $i=1$ to $\kappa$ do

13.

$$
K_{3}=K_{4} \leftarrow\lceil r / 10\rceil \text {-core of } G_{3}, G_{4}, \Gamma_{j} \leftarrow G_{j}\left[K_{j}\right], j=3,4 \text {. }
$$

14.

if $\hat{a}_{i} \notin K_{3}$ then

15.

Execute ConNectBACK $\left(K_{3}, \hat{a}_{i}, \hat{a}_{i}^{\prime}, i, W_{C B}^{(3)}\right) \mathrm{fi}$

16.

if $\hat{b}_{i} \notin K_{4}$ then

17.

Execute ConnectBack $\left(K_{4}, \hat{b}_{i}, \hat{b}_{i}^{\prime}, i, W_{C B}^{(4)}\right) \mathbf{f i}$

18. if $i \notin L$ then

19.

Construct random walk $W_{i}^{(4)}$ starting at $\hat{b}_{i}^{\prime}$ in $\Gamma_{4}$, the endpoint is denoted by $b_{i}^{*}$.

20. Execute WALK $\left(\hat{a}_{i}^{\prime}, \hat{b}_{i}^{\prime}, b_{i}^{*}, \Gamma_{3}, \Gamma_{4}\right), W_{i}^{(3)} \leftarrow \hat{W}_{l}$.

21. $G_{3} \leftarrow G_{3} \backslash E\left(\hat{W}_{j}\right), j=1, \ldots, l ; G_{4} \leftarrow G_{4} \backslash E\left(W_{i}^{(4)}\right)$

22.

23. $W_{i}^{(3)} \leftarrow\left(W_{C B}^{(3)}, W_{i}^{(3)}\right), W_{i}^{(4)} \leftarrow\left(W_{C B}^{(4)}, W_{i}^{(4)}\right)$.

24.

fi

od

25. end GenPaths

\subsubsection{Subroutine CONNECTBACK.}

The purpose of ConNECTBACK is to connect a vertex $x$ to vertices of large degree. All walks are done on vertices of large degree and in Steps 3-6 we check that the start point $x$ has large degree in $\Gamma_{5}$. ( $\Gamma_{5}$ is initially $G_{5}$ but loses vertices and edges as the algorithm proceeds). If not, we put it into $L$ for later. We do an edge avoiding random walk $W_{C B}$ from $x$ until a vertex $x^{\prime}$ of $K$ is reached. Later we replace $x$ by $x^{\prime}$. By edge avoiding, we mean that one is not allowed to use the same edge more than once. We do this for technical reasons, but it does not seem unreasonable anyway. This is done in Steps 7-10. 
1. subroutine CONNECTBACK $\left(K, x, x^{\prime}, i, W_{C B}\right)$

2. begin

3. $\quad \Gamma_{5} \leftarrow\lceil r / 10\rceil$-core of $G_{5}$

4. $\quad$ if $x \notin \Gamma_{5}$ then

5. $L \leftarrow L \cup\{i\}$

6. $\quad$ exit

7. $\quad$ fi

8. Construct an edge avoiding random walk $W_{C B}$ starting at $x$ in $\Gamma_{5}$ until $W_{C B}$ visits some vertex $x^{\prime}$ in $K$

9. (This is a trivial walk if $x \in K$ ).

10. $G_{5} \leftarrow G_{5} \backslash W_{C B}$

11. end CONNECTBACK

\subsubsection{Subroutine WALK.}

To connect $\hat{a}_{i}$ and $\hat{b}_{i}$ after Phase 2(a) we first connect them (if necessary) to the $\lceil r / 10\rceil$-core of $G_{3}, G_{4}$. We then do a random walk $W_{i}^{(4)}$ in $\Gamma_{4}$ starting at $\hat{b}_{i}^{\prime}$ and ending at $b_{i}^{*}$. We then try to join $\hat{a}_{i}^{\prime}$ to $b_{i}^{*}$.

To join $\hat{a}_{i}^{\prime}$ and $b_{i}^{*}$, the reader might expect us to choose a random walk from those with endpoints $\hat{a}_{i}^{\prime}, b_{i}^{*}$. The main problem with this is that the distribution of $b_{i}^{*}$ may be significantly different from the steady state distribution of a walk from $\hat{a}_{i}^{\prime}$ in $\Gamma_{3}$. If we choose a walk in this manner then deleting it will condition the graph in a way which is complex to analyze, especially as we have to repeat the procedure $\kappa$ times.

We overcome this by choosing a set of random walks and use rejection sampling to make the final walk have the correct distribution. There is still the complication that the $b_{i}^{*}$ are chosen before we do the walks. This leads to the subroutine WALK described next. $\operatorname{WALK}\left(\hat{a}_{i}^{\prime}, \hat{b}_{i}^{\prime}, b_{i}^{*}, \Gamma_{3}, \Gamma_{4}\right)$ generates a series of random walks of length $\tau_{0}$ in $\Gamma_{3}$ starting from $\hat{a}_{i}^{\prime}$. The last walk generated ends at $b_{i}^{*}$ which has the distribution

$$
\hat{p}_{v}=P_{\Gamma_{4}}^{\left(\tau_{0}\right)}\left(\hat{b}_{i}^{\prime}, v\right)
$$

[There is the question as to why we do not try to construct a walk from $\hat{a}_{i}$ to $\hat{b}_{i}$. We need in WALK to generate an endpoint from the same distribution as the target. This will not be so easy if $\hat{b}_{i}$ has dropped out of the $[r / 10\rceil$-core.]

The somewhat strange method used to generate these walks will be further explained in Section 8. 
1. subroutine $\operatorname{WALK}\left(\hat{a}_{i}^{\prime}, \hat{b}_{i}^{\prime}, b_{i}^{*}, \Gamma_{3}, \Gamma_{4}\right)$

2. begin

3. $\quad p_{v} \leftarrow P_{\Gamma_{3}}^{\left(\tau_{0}\right)}\left(\hat{a}_{i}^{\prime}, v\right)$ for $v \in K_{3}$

4. $\quad \hat{p}_{v} \leftarrow P_{\Gamma_{4}}^{\left(\tau_{0}\right)}\left(\hat{b}_{i}^{\prime}, v\right)$ for $v \in K_{4}$ (the distribution of $b_{i}^{*}$.)

5. $\quad p_{\min } \leftarrow \min \left\{p_{v}: v \in K_{3}\right\}$

6. $\quad \hat{p}_{\max } \leftarrow \max \left\{\hat{p}_{v}: v \in K_{4}\right\}$

7. Choose $l$ from the geometric distribution with probability of success $s=p_{\min } / \hat{p}_{\max }$

8. $\quad$ for $k$ from 1 to $l-1$ do

9. Choose $x_{k}$ according to $\operatorname{Pr}\left(x_{k}=v\right)=\left(p_{v}-\hat{p}_{v} p_{\min } / \hat{p}_{\max }\right) /(1-s)$

10. od

11. $\quad x_{l} \leftarrow b_{i}^{*}$

12. $\quad$ for $k$ from 1 to $l$ do

13. Pick a walk $\hat{W}_{k}$ of length $\tau_{0}$ in $\Gamma_{3}$ according to the distribution on trajectories, conditioned on start point $=\hat{a}_{i}^{\prime}$ and end point $=x_{k}$

14. od

15. output $\hat{W}_{1}, \hat{W}_{2}, \ldots, \hat{W}_{l}$

16. end WALK

The distributions $p_{v}$ and $\hat{p}_{v}$ can be computed in $O\left(n m \tau_{0}\right)$ time by computing powers of the transition matrix, after which a random walk can be found in $O\left(n \tau_{0}\right)$ time. (For details see [10].) The analysis will show that in the range of interest, whp, $s$ is bounded away from zero by a constant, hence the expected total ruming time of WALK is $O\left(n m \tau_{0}\right)$.

\subsection{Phase 3.}

There is still the set $L$ of pairs which have not been connected by paths. We will show later that whp $|L|$ is at most $n /(\log n)^{C_{0}}$. As such, these pairs can be dealt with by the algorithm of [10], using graph $G_{6}$.

\section{Configuration model}

\subsection{Model.}

We first show (Lemma 2 below) that the graphs $G_{i}, i=2, \ldots, 6$ are random given their degree sequences.

The simplest model for graphs with a fixed degree sequence is the configuration model of Bollobás [4] which is a probabilistic interpretation of the counting formula of Bender and Canfield [2]. Let $\mathbf{d}=\left\{d_{1}, d_{2}, \ldots, d_{\nu}\right\}$ denote a degree sequence, block $D_{i}=\left\{1, \ldots, d_{i}\right\} \times\{i\}$ for $1 \leq i \leq \nu$ and $D=\cup_{i=1}^{\nu} D_{i}$ be the set of points. Let $\Omega=\Omega(D)$ be the set of partitions of $D$ into pairs. Such partitions are called configurations. If $F \in \Omega$ then the multigraph $M(F)$ is defined as follows: $V(M)=[\nu]$ and there is an edge $\{i, j\}$ for every pair in $F$ of the form $\{(x, i),(y, j)\}$ (for some $x$ and $y$ ). Assume $F$ is chosen randomly from $\Omega$. The salient properties of $M=M(F)$ (see [4]) are: 
Lemma 1 (a) If $M$ is simple, then it is equally likely to be any simple graph with degree sequence $\mathbf{d}$.

(b) $\operatorname{Pr}(M$ is simple $)=\exp \left\{-O\left(\mu^{2} / \nu^{2}\right)\right\}$ where $\mu=|D| / 2$ is the number of edges in $M-$ hence $2 \mu / \nu$ is the average degree of $M$.

It therefore suffices to replace $G_{n, r}$ by a random configuration multigraph $G=M(F)$ where $\mathbf{d}=(r, r, \ldots, r)$. The corresponding random partition is denoted by $F_{0}$. Any property that holds whp in this model, holds whp in $G_{n, r}$ since $\exp \left\{-O\left(\mu^{2} / \nu^{2}\right)\right\}=e^{-O(1)}$. In what follows the graphs $G_{2}, \ldots, G_{6}$ are multigraphs associated with configurations $F_{2}, \ldots, F_{6}$.

\subsection{Random walks on configurations.}

To analyse random walks on the graphs $G_{i}$, we need to be sure they have the correct distribution.

Lemma 2 For each $i=2, \ldots, 6$ the configuration $F_{i}$ produced by algorithm SPLIT is random given its degree sequence i.e. conditional on having degree sequence $\mathbf{d}, F_{i}$ is equally likely to be any pairing of the corresponding $\sum d_{i}$ points.

Proof: Let $F^{\prime}$ be obtained from $F$ by including each pair of $F$ with probability $1 / 6$. We first show that $F^{\prime}$ is a random configuration given its degree sequence. Notice that

$$
\operatorname{Pr}\left(F^{\prime} \mid \mathrm{d}\right)=\frac{\operatorname{Pr}\left(F^{\prime}\right)}{\operatorname{Pr}(\mathrm{d})}=\frac{\sum_{F} \operatorname{Pr}\left(F^{\prime} \mid F\right)}{|\Omega| \operatorname{Pr}(\mathrm{d})}
$$

and $\operatorname{Pr}\left(F^{\prime} \mid F\right)=(1 / 6)^{\left(\sum d_{i}\right) / 2}(5 / 6)^{\left(r n-\sum d_{i}\right) / 2}$ if $F^{\prime} \subset F$ and 0 otherwise. Suppose $F_{1}$ and $F_{2}$ are two configurations with the same degree sequence $\mathbf{d}$, and let $\mathcal{F}_{1}=\left\{F \mid F_{1} \subset F\right\}$ and $\mathcal{F}_{2}=\left\{F \mid F_{2} \subset F\right\}$. It suffices to show that $\left|\mathcal{F}_{1}\right|=\left|\mathcal{F}_{2}\right|$. Let $E_{0}$ be the set of pairs $F_{1} \oplus F_{2}=\left(F_{1} \backslash F_{2}\right) \cup\left(F_{2} \backslash F_{1}\right)$. Define mapping $f: \mathcal{F}_{1} \rightarrow \mathcal{F}_{2}$ as follows: Given $F \in \mathcal{F}_{1}$, let $f(F)=\left[F \backslash\left(E_{0} \cap F_{1}\right)\right] \cup\left(E_{0} \cap F_{2}\right)$. It's easy to see that $f(F) \in \mathcal{F}_{2}$ and $f^{-1}: \mathcal{F}_{2} \rightarrow \mathcal{F}_{1}$ is well-defined as $f^{-1}(F)=\left[F \backslash\left(E_{0} \cap F_{2}\right)\right] \cup\left(E_{0} \cap F_{1}\right)$. Hence $f$ is a bijection and $\left|\mathcal{F}_{1}\right|=\left|\mathcal{F}_{2}\right|$.

Thus (in an obvious notation) $F_{1}^{\prime}, \ldots, F_{6}^{\prime}$ are all random configurations. Deleting a set $D_{j}$ and all $t$ pairs containing a member of $D_{j}$ from $F_{i}^{\prime}$ preserves randomness since every derived configuration arises from the same number of possible configurations $F_{i}^{\prime}$. So the final configurations $F_{2}, \ldots, F_{6}$ are random, given their degree sequences.

We need to show that removing the edges of a random walk does not condition the pairings of the remaining points. We now consider the construction of a random configuration $F$. It is useful to think of $F$ as being constructed sequentially.

1. Algorithm Construct

2. begin

3. $\quad F_{0} \leftarrow \emptyset ; R_{0} \leftarrow W$

4. $\quad$ for $t=1$ to $|W| / 2$ do

5. $\quad$ Choose $u_{t} \in R_{t-1}$ arbitrarily

6. Choose $v_{t}$ randomly from $R_{t-1} \backslash\left\{u_{t}\right\}$

7. $\quad F_{t} \leftarrow F_{t-1} \cup\left\{\left\{u_{t}, v_{t}\right\}\right\} ; R_{t} \leftarrow R_{t-1} \backslash\left\{u_{t}, v_{t}\right\}$

$8 . \quad$ od

9. output $F$

10. end Construct 
It is important to observe that for any $t>0, F \backslash F_{t}$ is a random member of $\Omega\left(R_{t}\right)$.

An important consequence of the above observation is that if we start with $M=M(F)$, then the multigraph obtained by removing from $M$ the edges of a random walk $W$ remains unconditioned. Indeed, we may imagine ConstRUCT as performed in parallel with our walk $W$. Suppose our walk makes a transition from a vertex $x$ and the current value of $R_{t}$ in ConstruCt is $R$. The transition from $x$ is equivalent to choosing a random member $u=u_{t}$ of $D_{x}$. If $u \in R$, then we perform one step of ConsTRUCT and pair $u$ with a point $v=v_{t} \in R \backslash\{u\}$. If $v \in D_{y}$ for some $y$, then the walk makes a transition from $x$ to $y$. If $u \notin R$ then $v$ is the point already paired with $u$. Thus, since $F \backslash F_{t}$ is random, we see that removing from $M$ the edges of a random walk results in a multigraph from a random configuration. The same argument can be applied to the edge avoiding walk of ConNECTBACK. In the latter case we do not need to consider the case $u \notin R_{t}$.

In summary, we can conduct our analysis of Phase 3 always assuming that the graphs $G_{2}$ - $G_{5}$ are random configuration multigraphs, given their degree sequences.

\section{Analysis of Phase 1}

We begin with some discussion of $G$ :

Lemma 3 Whp every subgraph $H$ of $G$ which is induced by a set of vertices $S,|S| \leq n_{0}=$ $27 n /\left(8 e^{6} r^{3}\right)$, has at most $3|S| / 2$ edges.

Proof: Let $D_{S}=\bigcup_{i \in S} D_{i}=\left\{w_{1}, w_{2}, \ldots, w_{r|S|}\right\}$. Let us randomly pair up $w_{1}, w_{2}, \ldots$ At any stage, if $w_{i}$ has not been paired with any of $w_{1}, w_{2}, \ldots, w_{i-1}$, and given the pairings of these points, the probability that $w_{i}$ is paired with something in $D_{S}$ is at most $|S| / n$. Hence

$$
\begin{aligned}
\operatorname{Pr}\left(\exists S:|S|=s \leq n_{0}, S \text { contains 3s/2 edges }\right) & \leq \sum_{s=4}^{n_{0}}\left(\begin{array}{c}
n \\
s
\end{array}\right)\left(\begin{array}{c}
r s \\
3 s / 2
\end{array}\right)\left(\frac{s}{n}\right)^{3 s / 2} \\
& \leq \sum_{s=4}^{n_{0}}\left(\frac{n e}{s}\right)^{s}\left(\frac{2 e r s}{3 n}\right)^{3 s / 2} \\
& =\sum_{s=4}^{n_{0}}\left(\frac{8 e^{5} r^{3} s}{27 n}\right)^{s / 2} \\
& =O\left(n^{-2}\right) .
\end{aligned}
$$

The next lemma concerns the size of $K$ at the end of SPLIT.

Lemma 4 If $K$ is as defined in algorithm SPLIT then whp

$$
|K| \geq\left(1-e^{-r / 800}\right) n .
$$

Proof: Fix $1 \leq i \leq 6$ and consider $G_{i}^{\prime}$ immediately after Step 4. If $v \in V$ then $d_{G_{i}^{\prime}}(v)$ has distribution $\operatorname{Bin}(r, 1 / 6)$ and hence at this point (1) implies

$$
\operatorname{Pr}\left(d_{G_{i}^{\prime}}(v) \leq\lfloor r / 7\rfloor\right) \leq \epsilon_{1}=e^{-r / 600} .
$$

So if $V_{i}=\left\{v \in V: d_{G_{i}^{\prime}}(v)>\lfloor r / 7\rfloor\right\}$ then $\mathbf{E}\left(\left|V_{i}\right|\right) \geq\left(1-\epsilon_{1}\right) n$. Now the random variable $\left|V_{i}\right|$ is determined by the outcome of $r n / 2$ random choices. Also, changing one such choice results 
in changing $\left|V_{i}\right|$ by at most 2. Applying the Azuma-Hoeffding martingale tail inequality ([5], [21]) we obtain

$$
\operatorname{Pr}\left(\left|V_{i}\right| \leq \mathbf{E}\left(\left|V_{i}\right|\right)-t\right) \leq e^{-t^{2} /(r n)}
$$

Putting $t=\epsilon_{1} n$ we see that whp

$$
\left|V_{i}\right| \geq\left(1-2 \epsilon_{1}\right) n, \quad 1 \leq i \leq 6 .
$$

Now consider $K$ at the end of Step 5. Let $W_{0}=V \backslash V_{1}$ and define a sequence $w_{1}, w_{2}, \ldots, w_{k}$ as follows: Suppose $W_{j}=W_{0} \cup\left\{w_{1}, w_{2}, \ldots, w_{j}\right\}$ and $w_{j+1}$ is an arbitrary vertex not in $W_{j}$ but which has at least 2 neighbours in $W_{j}$. The sequence stops at index $k$ when every vertex not in $W_{k}$ has at most one neighbour in $W_{k}$. Now every vertex not in $W_{k}$ has $d_{G_{i}^{\prime}}(v)>\lfloor r / 7\rfloor$ and hence has degree at least $\lfloor r / 7\rfloor$ in $V \backslash W_{k}$. Thus $|K| \geq n-\left|W_{k}\right|$. We now show that whp $k<6 \epsilon_{1} n$. If not then

- $W_{6 \epsilon_{1} n}$ has at most $8 \epsilon_{1} n$ vertices.

- $W_{6 \epsilon_{1} n}$ contains at least $12 \epsilon_{1} n$ edges.

For sufficiently large $r$, this contradicts the conclusion of Lemma 3. Thus whp, at the end of Step 5,

$$
|K| \geq\left(1-8 \epsilon_{1}\right) n
$$

Now consider the execution of Step 7. One can see that the value for $K$ at the termination of this step is independent of the order in which vertices are deleted by the $k$-core algorithm. So assume that we start by deleting $\bigcup_{i=2}^{6}\left(V \backslash V_{i}\right)$. (4) and (5) imply that whp after this $|K| \geq\left(1-18 \epsilon_{1}\right) n$. We then argue, similarly to above, that no more than $72 \epsilon_{1} n$ more vertices will be deleted by the $k$-core algorithm. The lemma follows for sufficiently large $r$.

\section{Analysis of Phase 2}

In this section we show that if our input graph $G=(V, E)$ is $G_{n, r}$ then whp, after we run SPLIT, we can find in $G_{1}$ edge-disjoint paths from $a_{i}$ to $\tilde{a}_{i}$, and $b_{i}$ to $\tilde{b}_{i}$, for $1 \leq i \leq \kappa$, for any choice of $a_{1}, \ldots, b_{\kappa}$ consistent with the premises of Theorem 1 , and every choice for $\tilde{a}_{1}, \ldots, \tilde{b}_{\kappa}$.

Let $A$ and $Z$ be as defined in Section 4.2. For $S \subseteq V$, let

$$
\alpha(S)=\mid\left\{i: a_{i} \text { or } b_{i} \in S\right\} \mid \text { and } \xi(S)=|S \cap Z| .
$$

For sets $S, T \subseteq V$, let $e_{G_{1}}(S, T)$ denote the number of edges of $G_{1}$ with an endpoint in $S$ and the other endpoint in $T$. It suffices to prove that

$$
e_{G_{1}}(S, \bar{S}) \geq \xi(\bar{S})-\alpha(\bar{S}), \quad \forall S \subseteq V .
$$

We can then apply a theorem of Gale [14] (see Bondy and Murty [7], Theorem 11.8) to deduce the existence of the required flow in $G_{1}$ for the successful run of Phase 2.

Lemma 5 Whp $e_{G_{1}}(S, \bar{S}) \geq r|S| / 288$ for all $S \subseteq V,|S| \leq n / 2$. 
Proof: Assume first that $|S| \leq n_{0}$. From Lemma 3, we obtain

$$
e_{G}(S, \bar{S}) \geq\left(\delta\left(G_{1}\right)-3\right)|S| \geq(\lceil r / 7\rceil-3)|S| .
$$

So now assume $|S|>n_{0}$ and first consider $e_{G}(S, \bar{S})$. We claim that in the configuration model this stochastically dominates $\operatorname{Bin}(r|S| / 4,1 / 3)$. Apply algorithm ConstRUCT, always choosing $u_{t} \in D_{v}, v \in S$ whenever possible. This is definitely possible in the first $r|S| / 4$ choices. For each such choice, the probability that $v_{t} \in D_{w}, w \in \bar{S}$ is at least $1 / 3$, since $\left|R_{t-1} \cap S\right| \leq r|S| \leq r n / 2$ and $\left|R_{t-1} \cap \bar{S}\right| \geq r n / 4$. But by (1) with $\epsilon=1 / 2$,

$$
\operatorname{Pr}\left(\operatorname{Bin}\left(\frac{r|S|}{4}, \frac{1}{3}\right) \leq \frac{r|S|}{24}\right) \leq e^{-r|S| / 96}
$$

Thus

$$
\begin{aligned}
\operatorname{Pr}\left(\exists S: n_{0} \leq|S| \leq n / 2 \text { and } e_{G}(S, \bar{S}) \leq \frac{r|S|}{24}\right) & \leq \sum_{s=n_{0}}^{n / 2}\left(\begin{array}{c}
n \\
s
\end{array}\right) e^{-r s / 96} \\
& \leq \sum_{s=n_{0}}^{n / 2}\left(\frac{n e}{s} e^{-r / 96}\right)^{s} \\
& =o(1)
\end{aligned}
$$

for $r$ sufficiently large.

Assume then that $e_{G}(S, \bar{S}) \geq r|S| / 24$ for $n_{0} \leq|S| \leq n / 2$. Given this, $e_{G_{1}}(S, \bar{S})$ stochastically dominates $\operatorname{Bin}(r|S| / 24,1 / 6)$. Applying (1) again with $\epsilon=1 / 2$,

$$
\begin{aligned}
\operatorname{Pr}\left(\exists S: n_{0} \leq|S| \leq n / 2 \text { and } e_{G}(S, \bar{S}) \leq \frac{r|S|}{288}\right) & \leq \sum_{s=n_{0}}^{n / 2}\left(\begin{array}{l}
n \\
s
\end{array}\right) e^{-r s / 1152} \\
& \leq \sum_{s=n_{0}}^{n / 2}\left(\frac{n e}{s} e^{-r / 1152}\right)^{s} \\
& =o(1)
\end{aligned}
$$

for $r$ sufficiently large.

Now

$$
\alpha(\bar{S})=2 \kappa-\alpha(S) \geq 2 \kappa-\beta r|S|
$$

and so

$$
\xi(\bar{S})-\alpha(\bar{S}) \leq|Z \cap \bar{S}|-2 \kappa+\beta r|S| \leq \beta r|S|
$$

Thus Lemma 5 verifies (6) for $|S| \leq n / 2$ provided we choose $\beta<1 / 288$. For $|S|>n / 2$ we use

$$
e_{G_{1}}(S, \bar{S})=e_{G_{1}}(\bar{S}, S) \geq r|\bar{S}| / 288 \geq|Z \cap \bar{S}| \geq|Z \cap \bar{S}|-2 \kappa+\alpha(S)=\xi(\bar{S})-\alpha(\bar{S})
$$

and so Phase 2 succeeds whp. 


\section{Analysis of Phase 3}

\subsection{Construction of $W_{i}^{(2)}, W_{i}^{(5)}$}

For $v \in K$, let $d_{j, v}$ denote its degree in $\Gamma_{j}, j=2,3,4$ and let $\pi_{j, v}=d_{j, v} / \sum_{w \in K} d_{j, w}$ be the steady state distribution of a random walk on $\Gamma_{j}$. These values change as the algorithm progresses, but immediately prior to any random walk in a $\Gamma_{j}$ we ensure that

$$
\frac{r}{10} \leq d_{j, v} \leq \frac{2 r}{7}
$$

Hence $v \in K$ implies

$$
\frac{1}{3|K|} \leq \pi_{j, v} \leq \frac{3}{|K|}
$$

Let $\Gamma^{(i)}$ denote $\Gamma_{2}$ immediately prior to constructing the random walk starting at $z_{i}$ and let $K^{(i)}$ be its vertex set, the current value of $K$. Let $P^{(i)}$ denote the transition probability matrix of a random walk on $\Gamma^{(i)}$. Let $\lambda^{(i)}$ be the second largest eigenvalue of $P^{(i)}$. It is well known that the second eigenvalue determines the rate of convergence of a Markov chain to its steady state. An explicit form of this result was obtained by Jerrum and Sinclair [25]: if $P_{\Gamma^{(i)}}^{(t)}(u, v)$ denotes the probability that a random walk of length $t$ in $\Gamma^{(i)}$ which starts at $u$ will end at $v$, then

$$
\left|P_{\Gamma^{(i)}}^{(t)}(u, v)-\pi_{v}^{(i)}\right| \leq\left(\lambda^{(i)}\right)^{t} \sqrt{\frac{\pi_{v}^{(i)}}{\pi_{u}^{(i)}}} .
$$

We will argue later that whp throughout the algorithm

$$
|K| \geq\left(1-\epsilon_{K}\right) n
$$

where

$$
\epsilon_{K}=8 e^{-r / 250000} \text {. }
$$

We will argue that as long as $K$ is this large, vertices are being removed at a sufficiently slow rate so that the overall loss from $K$ is less than $\epsilon_{K} n$ whp. Thus the specific assumption at each stage is that (10) holds at the present time. We do not in this way "condition on the future".

We argued in Section 5.2 that $\Gamma^{(i)}$ is random, given its degree sequence. According to Theorem 2 of [8], (which is adapted from [16]) the second largest eigenvalue $\lambda^{(i)}$ is with probability $1-O\left(|K|^{-a}\right)=1-O\left(n^{-a}\right)$ bounded by $\gamma / \sqrt{r}$, where $\gamma$ depends only on $a$ and a bound on the ratio of the maximum and the minimum degrees of $\Gamma^{(i)}$, which we can take to be 3 by (7). Taking $a=4$ we have that $\gamma$ is a constant independent of $r$. Using this in (9) we obtain that conditional on an event of probability $1-O\left(n^{-4}\right)$

$$
\left|P_{\Gamma^{(i)}}^{(t)}(u, v)-\pi_{v}^{(i)}\right| \leq \frac{3 \gamma^{t}}{r^{t / 2}}
$$

So from (3) we see that for large $r$ we have

$$
\left|P_{\Gamma^{(i)}}^{\left(\tau_{0}\right)}(u, v)-\pi_{v}^{(i)}\right| \leq n^{-4}
$$

Let $\mathcal{E}^{(i)}$ be the intersection of the high probability events described in Lemmas 3,4 and 5 plus $(10),(12)$ for $\Gamma^{(1)}, \ldots, \Gamma^{(i)}$. We prove by induction on $i$ that

$$
\operatorname{Pr}\left(\mathcal{E}^{(i+1)} \mid \mathcal{E}^{(i)}\right) \geq 1-O\left(n^{-3}\right) .
$$


This is clearly true for $i=0$ and all we need to show is that (10) holds with the correct probability.

Fix $1 \leq i \leq \kappa$, let $s=100 \ln n$ and $\hat{V}_{2}$ be the set of vertices in $G_{2}$ which are incident with at least $r / 30$ edges of $W_{1}, \ldots, W_{i}$. For $S \subseteq K^{(0)}$ of size $s$, let $\mathcal{B}_{S}$ be the event that $S \subseteq \hat{V}_{2}$. We first show that for all such $S$,

$$
\operatorname{Pr}\left(\mathcal{B}_{S} \mid \mathcal{E}^{(i)}\right) \leq 2 e^{-r s / 250000}
$$

Let $S=\left\{v_{1}, \ldots, v_{s}\right\} \subset K^{(0)}$. Let $\mathcal{P}=\mathcal{P}(S)$ be the event that there exist $w \in K^{(1)} \backslash S$ and $t=r / 2000$ distinct paths $P_{1}, P_{2}, \ldots, P_{t}$ from $w$ to $S$ of length $\leq k_{0}=\left(\log _{r} n\right) / 10$. If such paths exist then the graph induced by $w, S, P_{1}, P_{2}, \ldots, P_{t}$ contains $s+u$ vertices and $\geq s+u+t-1$ edges for some $1 \leq u \leq\left(k_{0}-1\right) t+1$. This is because the addition of each new path to $S, w$ adds at least one more new edge than vertex. Thus,

$$
\begin{aligned}
\operatorname{Pr}(\mathcal{P}) & \leq \sum_{u=1}^{\left(k_{0}-1\right) t+1}\left(\begin{array}{c}
n \\
s+u
\end{array}\right)\left(\begin{array}{c}
2 r(s+u) / 7 \\
s+u+t-1
\end{array}\right)\left(\frac{3(s+u)}{n}\right)^{s+u+t-1} \\
& \leq \sum_{u=1}^{\left(k_{0}-1\right) t+1}\left(\frac{n e}{s+u}\right)^{s+u}\left(\frac{r(s+u) e}{n}\right)^{s+u+t-1} \\
& \leq \sum_{u=1}^{\left(k_{0}-1\right) t+1} \frac{\left(r e^{2}\right)^{s+u+t}(s+u)^{t-1}}{n^{t-1}} \\
& \leq \frac{2\left(r e^{2}\right)^{s+k_{0} t+1}\left(s+k_{0} t\right)^{t-1}}{n^{t-1}} \\
& \leq n^{-r / 2500} \\
& =e^{-r s / 250000}
\end{aligned}
$$

Explanation of (15): working within the configuration model we choose the $s+u$ vertices in at most $\left(\begin{array}{c}n \\ s+u\end{array}\right)$ ways. The corresponding blocks contain at most $2 r(s+u) / 7$ points $X$. We then choose the set of lowest indices $I$ of the $s+u+t-1$ pairs in at most $\left(\begin{array}{c}2 r(s+u) / 7 \\ s+u+t-1\end{array}\right)$ ways. The last factor is an upper bound on the probability that each point in $I$ chooses a partner in $X$.

Assume $\mathcal{P}$ does not occur. Let $W_{i}$ denote the walk $W_{i}^{(2)}$. Let $Z_{i, S}$ denote the number of edges incident with vertices in $S$ that are used by $W_{i}$ and let $N_{i, S}$ be the number of visits to $S$ by $W_{i}$. Consider $\rho_{S}$, the probability that a random walk $W$ from $u \in S$ returns to $S$ within $\tau_{0}$ steps.

Lemma 6 If $\mathcal{P}$ does not occur then $\rho_{S} \leq 1 / 20$.

Proof: Let $\operatorname{dist}(v, S)$ denote the length of a shortest path in $\Gamma^{(i)}$ from $v$ to $S$. Define $S_{j}=$ $\left\{v \in K^{(i)} \backslash S: \operatorname{dist}(v, S)=j\right\}$ for $j=1, \ldots, k_{0}-1$ and $S_{k_{0}}=\left\{v \in K^{(i)} \backslash S: \operatorname{dist}(v, S) \geq k_{0}\right\}$.

We define a random walk $X_{0}, X_{1}, \ldots$, where $X_{0}=0$ and $X_{t}=j$ iff $W$ is at a vertex $v \in S_{j}$ after $t$ steps $\left(S_{0}=S\right)$. Then given $\overline{\mathcal{P}}$ and that the minimum degree in $\Gamma^{(i)}$ is $r / 10$, for a random walk $W$,

$$
\begin{aligned}
& \operatorname{Pr}\left(X_{t}=j+1 \mid X_{t}=j\right) \geq 199 / 200, \quad j=1, \ldots, k_{0}-1 \\
& \operatorname{Pr}\left(X_{t+1}=k_{0} \mid X_{t}=k_{0}\right) \geq 199 / 200 .
\end{aligned}
$$


Now define another random walk $\tilde{X}_{0}=0, \tilde{X}_{1}, \ldots$, where

$$
\operatorname{Pr}\left(\tilde{X}_{t+1}=\tilde{X}_{t}+\delta\right)=\left\{\begin{array}{lll}
\frac{199}{200} & \delta=1 \\
\frac{1}{200} & \delta=-1 & \tilde{X}_{t}>0, \text { or } \\
& \delta=0 & \tilde{X}_{t}=0
\end{array}\right.
$$

Now for $u>0$ we have

$$
\operatorname{Pr}\left(\tilde{X}_{u}=0\right) \leq\left(\begin{array}{c}
u \\
\lceil u / 2\rceil
\end{array}\right)\left(\frac{1}{200}\right)^{\lceil u / 2\rceil} \leq 50^{-\lceil u / 2\rceil}
$$

since if $\tilde{X}_{u}=0$ at least $\lceil u / 2\rceil$ of the first $u$ moves must be to the left i.e. $\delta \leq 0$. So,

$$
\operatorname{Pr}\left(\exists u>0: X_{u}=0\right) \leq \sum_{u=1}^{\infty} 50^{-\lceil u / 2\rceil} \leq 2 \sum_{u=1}^{\infty} 50^{-u}=\frac{2}{49}
$$

It follows from (17) and (18) that $\tilde{X}_{j} \leq X_{j}$ in distribution up to the first time $u_{0}$ that $X_{u}=k_{0}$. So

$$
\operatorname{Pr}\left(\exists 0<u \leq u_{0}: X_{u}=0\right) \leq \frac{2}{49} .
$$

Next observe that for any $u>0$

$$
\operatorname{Pr}\left(X_{u+\frac{k_{0}}{10}}<\frac{k_{0}}{2} \mid X_{u} \geq \frac{k_{0}}{2}\right) \leq 2^{k_{0} / 10} 200^{-k_{0} / 20}=50^{-k_{0} / 20}
$$

since in order that $X_{u+\frac{k_{0}}{10}}<\frac{k_{0}}{2}$ there must be at least $\frac{k_{0}}{20}$ moves to the left.

Putting $u_{i}=u_{0}+i \frac{k_{0}}{10}$ we see that

$$
\operatorname{Pr}\left(\exists i, 1 \leq i \leq 10: X_{u_{i}}<\frac{k_{0}}{2}\right) \leq 10 \times 50^{-k_{0} / 20} .
$$

Then observe that if $X_{u}=0$ for some $u_{0}<u \leq \tau_{0}$ then we must have $u_{i}<\frac{k_{0}}{2}$ for some $1 \leq i \leq 10$. This together with (19) completes the proof of the lemma.

Let $q_{k}=\operatorname{Pr}\left(N_{i, S}=k \mid \mathcal{E}^{(i)}\right)$ for $k \geq 1$. We claim that given $W_{1}, W_{2}, \ldots, W_{i-1}$,

$$
q_{k}<\left(\frac{1000 s \log _{r} n}{n}\right) 20^{-(k-1)}
$$

To prove (20) for $k=1$, fix $\Gamma^{(i)}$ and let $h_{v}(t)$ be the probability that the walk is at $v$ after $t$ steps. For $t=0$, we have two cases.

Case 1: Subroutine ConnectBack is not executed. In this case $W_{i}$ starts from $z_{i}$ which is a vertex chosen uniformly at random from $V_{2} \backslash\left\{z_{1}, z_{2}, \ldots, z_{i-1}\right\}$. Notice that the execution of Phase 2 does not depend on the paths created in Phase 1 . Therefore we can analyse Phase 2 without reference to Phase 1 and it is legitimate to consider $z_{i}$ as chosen randomly in this way, independent of the state of $\Gamma^{(i)}$. So

$$
h_{v}(0) \leq \frac{2}{\left|V_{2}\right|} \leq 10 \pi_{v}^{(i)} .
$$


Case 2: Subroutine ConnectBACK is executed. In this case $W_{i}$ starts from $z_{I}^{\prime}$ which is the end point of a random walk in $\Gamma_{3}$. The random walks in $\Gamma_{3}$ only use unexposed edges. The endpoint is chosen randomly from $K^{(i)}$ with probability at most $2 \pi_{v}^{(i)}$, see Section 8.3.

So in either case $h_{v}(0) \leq 10 \pi_{v}^{(i)}$. We can now inductively show that $h_{v}(t) \leq 10 \pi_{v}^{(i)}$ for all $t \geq 0$. Using the stationarity equations, we have

$$
h_{v}(t+1)=\sum_{w \in N_{\Gamma^{(i)}}(v)} \frac{h_{w}(t)}{d_{w}^{(i)}} \leq 10 \sum_{w \in N_{\Gamma^{(i)}}(v)} \frac{\pi_{w}^{(i)}}{d_{w}^{(i)}}=10 \pi_{v}^{(i)} \leq \frac{100}{n} .
$$

The last inequality follows from (8) and (10). Hence, if $\hat{q}_{1}$ denotes the probability that $N_{I, S} \geq 1$ then

$$
q_{1} \leq \hat{q}_{1} \leq \sum_{v \in S} \sum_{t=0}^{\tau_{0}} h_{v}(t) \leq \frac{1000 s \log _{r} n}{n} .
$$

For $k \geq 2,(20)$ follows from

$$
q_{k} \leq \rho_{S}^{k-1} \hat{q}_{1} .
$$

We prove next that whp the size of $\hat{V}_{2}$ is small. First (20) implies that

$$
\begin{aligned}
\mathbf{E}\left(e^{N_{i, S}} \mid \mathcal{E}^{(i)}, \overline{\mathcal{P}}\right) & \leq 1+\sum_{k \geq 1} e^{k}\left(\frac{1000 s \log _{r} n}{n}\right) \rho_{S}^{k-1} \\
& \leq 1+\frac{C_{2} s \log _{r} n}{n}
\end{aligned}
$$

Clearly $Z_{i, S} \leq 2 N_{i, S}$. Thus for any $t>0$,

$$
\begin{aligned}
& \operatorname{Pr}\left(\sum_{j=1}^{i} Z_{j, S} \geq s t \mid \mathcal{E}^{(i)}, \overline{\mathcal{P}}\right) \\
& \quad \leq e^{-s t / 2} \mathbf{E}\left(\exp \left\{\sum_{j=1}^{i} N_{j, S}\right\} \mid \mathcal{E}^{(i)}, \overline{\mathcal{P}}\right) \\
& \quad \leq e^{-s t / 2}\left(1+\frac{C_{2} s \log _{r} n}{n}\right) \mathbf{E}\left(\exp \left\{\sum_{j=1}^{i-1} N_{j, S}\right\} \mid \mathcal{E}^{(i)}, \overline{\mathcal{P}}\right) \\
& \quad \leq e^{-s t / 2} \exp \left\{\frac{C_{2} s \log _{r} n}{n}\right\} \mathbf{E}\left(\exp \left\{\sum_{j=1}^{i-1} N_{j, S}\right\} \mid \mathcal{E}^{(i-1)}, \overline{\mathcal{P}}\right) \frac{\operatorname{Pr}\left(\mathcal{E}^{(i-1)}, \overline{\mathcal{P}}\right)}{\operatorname{Pr}\left(\mathcal{E}^{(i)}, \overline{\mathcal{P}}\right)} \\
& \quad \leq \exp \left\{-\frac{s t}{2}+i \frac{C_{2} s \log _{r} n}{n}\right\} \frac{1}{\mathbf{P r}\left(\mathcal{E}^{(i)}, \overline{\mathcal{P}}\right)} \\
& \quad \leq 2 \exp \left\{-\frac{s t}{2}+2 \kappa \frac{C_{2} s \log _{r} n}{n}\right\} \\
& \quad \leq 2 \exp \left\{s\left(-t / 2+2 \alpha r C_{2}\right)\right\}
\end{aligned}
$$

Take $t=r / 30$ and $\alpha \leq\left(240 C_{2}\right)^{-1}$ so that $-t+2 \alpha C_{2} r<-r / 120$. Therefore

$$
\operatorname{Pr}\left(S \subseteq \hat{V}_{2} \mid \mathcal{E}^{(i)}, \overline{\mathcal{P}}\right) \leq 2 e^{-r s / 120} .
$$

Thus,

$$
\operatorname{Pr}\left(S \subseteq \hat{V}_{2} \mid \mathcal{E}^{(i)}\right) \leq \operatorname{Pr}\left(\mathcal{P} \mid \mathcal{E}^{(i)}\right)+\operatorname{Pr}\left(S \subseteq \hat{V}_{2} \mid \mathcal{E}^{(i)}, \overline{\mathcal{P}}\right) \leq 2 e^{-r s / 250000}
$$


proving (14).

So if $\epsilon_{2}=e^{-r / 250000}$ then it follows easily that whp $\left|\hat{V}_{2}\right| \leq 3 \epsilon_{2} n$. Indeed

$$
\begin{aligned}
\operatorname{Pr}\left(\left|\hat{V}_{2}\right| \geq 3 \epsilon_{2} n \mid \mathcal{E}^{(i)}\right) & =\operatorname{Pr}\left(\left(\begin{array}{c}
\hat{V}_{2} \mid \\
s
\end{array}\right) \geq\left(\begin{array}{c}
3 \epsilon_{2} n \\
s
\end{array}\right) \mid \mathcal{E}^{(i)}\right) \\
& \leq \frac{\mathbf{E}\left(\left(\begin{array}{c}
\hat{V}_{2} \mid \\
s
\end{array}\right) \mid \mathcal{E}^{(i)}\right)}{\left(\begin{array}{c}
3 \epsilon_{2} n \\
s
\end{array}\right)} \leq \frac{\left(\begin{array}{c}
n \\
s
\end{array}\right) 2 \epsilon_{2}{ }^{s}}{\left(\begin{array}{c}
3 \epsilon_{2} n \\
s
\end{array}\right)} \\
& \leq 2(e / 3)^{s}=O\left(n^{-9}\right) .
\end{aligned}
$$

Suppose that besides $\hat{V}_{2}$, we also delete $v_{1}, v_{2}, \ldots, v_{\ell}$ from $K_{2}$ in Step 4 of GenPATHs. We claim that $\ell \leq \epsilon_{2} n$. To see this note that $v_{i}$ must have at least $[r / 7\rceil-r / 30-r / 10 \geq r / 200$ neighbours in $\hat{V}_{2} \cup\left\{v_{1}, \ldots, v_{i-1}\right\}$. Thus $\hat{V}_{2} \cup\left\{v_{1}, \ldots, v_{i-1}\right\}$ has at least $\ell r / 200$ edges. This contradicts Lemma 3 for $\ell>\epsilon_{2} n$ and $r$ sufficiently large, which proves that $|K| \geq n\left(1-4 \epsilon_{2}\right)$ whp for the duration of Phase 2(a).

\subsection{Construction of $W_{i}^{(4)}$ and Analysis of WALK}

The arguments we gave above apply to constructing and deleting $W_{i}^{(4)}$ in $\Gamma_{4}$ and we will focus on discussing the subroutine WALK. Consider a modification of WALK defined as follows:

1. subroutine WALK1 $\left(\hat{a}_{i}^{\prime}, \hat{b}_{i}^{\prime}, b_{i}^{*}, \Gamma_{3}, \Gamma_{4}\right)$

2. begin

3. $\quad p_{v} \leftarrow P_{\Gamma_{3}}^{\left(\tau_{0}\right)}\left(\hat{a}_{i}^{\prime}, v\right)$ for $v \in K_{3}$

4. $\quad \hat{p}_{v} \leftarrow P_{\Gamma_{4}}^{\left(\tau_{0}\right)}\left(\hat{b}_{i}^{\prime}, v\right)$ for $v \in K_{4}$ (the distribution of $b_{i}^{*}$.)

5. $\quad p_{\min } \leftarrow \min \left\{p_{v}: v \in K_{3}\right\}$

6. $\quad \hat{p}_{\max } \leftarrow \max \left\{\hat{p}_{v}: v \in K_{4}\right\}$

7. $\hat{\ell} \leftarrow 0$

8. forever do

9. $\hat{\ell} \leftarrow \hat{\ell}+1$

10. $\quad$ Pick a walk $\bar{W}_{\hat{\ell}}$ of length $\tau_{0}$ in $K_{3}$ according to the distribution on trajectories, conditioned on start point $=\hat{a}_{i}^{\prime}$

11. Let $\hat{x}_{\hat{\ell}}$ be the terminal vertex of $\bar{W}_{\hat{\ell}}$

12. With probability $\hat{p}_{\hat{x}_{\hat{\ell}}} p_{\min } /\left(p_{\hat{x}_{\hat{\ell}}} \hat{p}_{\max }\right)$ accept $\bar{W}_{\hat{\ell}}$ and exitloop

13. od

14. output $\bar{W}_{1}, \bar{W}_{2}, \ldots, \bar{W}_{\hat{\ell}}$

15. end WALK1

Lemma 7 In WALK1, $\hat{x}_{\hat{\ell}}$ is chosen according to the distribution $\hat{p}$.

Proof: The probability $s$ that a walk is accepted at the last step in the loop is given by

$$
s=\sum_{v \in K_{3}} p_{v} \frac{\hat{p}_{v} p_{\min }}{p_{v} \hat{p}_{\max }}=\frac{p_{\min }}{\hat{p}_{\max }} .
$$


(Observe that $\hat{p}_{\max } \geq 1 /\left|K_{4}\right|=1 /\left|K_{3}\right| \geq p_{\min }$.) Thus

$$
\operatorname{Pr}\left(\hat{x}_{\hat{\ell}}=v\right)=\sum_{k=0}^{\infty}(1-s)^{k} p_{v} \frac{\hat{p}_{v} p_{\min }}{p_{v} \hat{p}_{\max }}=\hat{p}_{v} .
$$

Hence $\bar{W}_{\bar{r}}$ is a random walk to a vertex chosen with distribution $\hat{p}$. Furthermore, by (8),

$$
s \geq \sigma=\frac{1}{9}
$$

and therefore the expected number of walks generated is constant.

There is a minor problem in that we want to choose the endpoints before we do the walks. This leads to the algorithm WALK described before. We now turn to its analysis.

Lemma 8 Suppose that $b_{i}^{*}$ is chosen from $K_{3}$ with distribution $\hat{p}$. Then the walks $\bar{W}_{1}, \ldots, \bar{W}_{\hat{\ell}}$ in $\operatorname{WALK} 1\left(\hat{a}_{i}^{\prime}, \hat{b}_{i}^{\prime}, b_{i}^{*}, \Gamma_{3}, \Gamma_{4}\right)$, and the walks $\hat{W}_{1}, \ldots, \hat{W}_{\ell}$ in $\operatorname{WALK}\left(\hat{a}_{i}^{\prime}, \hat{b}_{i}^{\prime}, b_{i}^{*}, \Gamma_{3}, \Gamma_{4}\right)$ have the same distribution.

Proof: Note first from the proof of Lemma 7 that $\hat{\ell}$ and $\ell$ have the same truncated geometric distribution. Also, we have from Lemma 7 that $\hat{x}_{\hat{\ell}}$ and $x_{\ell}=b_{i}^{*}$ have the same distribution. Consider next that for $v_{1}, v_{2}, \ldots, v_{i} \in K_{3}$,

$$
\begin{aligned}
\operatorname{Pr}\left(\hat{x}_{1}\right. & \left.=v_{1}, \ldots, \hat{x}_{i}=v_{i} \text { and } \hat{\ell}>i\right) \\
& =\prod_{j=1}^{i}\left(\left(1-\frac{\hat{p}_{v_{j}} p_{\min }}{p_{v_{j}} \hat{p}_{\max }}\right) p_{v_{j}}\right)=\prod_{j=1}^{i}\left(p_{v_{j}}-\frac{\hat{p}_{v_{j}} p_{\min }}{\hat{p}_{\max }}\right) \\
& =(1-s)^{i} \prod_{j=1}^{i}\left(\frac{p_{v_{j}}-\hat{p}_{v_{j}} p_{\min } / \hat{p}_{\max }}{1-s}\right) \\
& =\operatorname{Pr}\left(x_{1}=v_{1}, \ldots, x_{i}=v_{i} \text { and } \ell>i\right) .
\end{aligned}
$$

Thus $\hat{x}_{1}, \hat{x}_{2}, \ldots, \hat{x}_{\hat{\ell}}$ and $x_{1}, x_{2}, \ldots, x_{\ell}$ have the same distribution. Finally, the lemma follows from the fact that the distribution of $\bar{W}_{j}$ conditional on $\hat{x}_{j}=v$ is clearly equal to that of $\hat{W}_{j}$ conditional on $x_{j}=v$.

Now we need to show that there are not many vertices in $G_{3}$ having degrees less that $r / 10$ after the $\ell$ walks are deleted. We shall refer to these walks as the $i$ th bundle, $B_{i}$. We shall closely follow the line of proof used in the analysis in Section 8.1, with all the events now referring to $\Gamma_{3}$ rather than $\Gamma_{2}$. As before, the proof reduces to showing that given $\mathcal{E}^{(I)}$, after the removal of the bundles $B_{1}, \ldots, B_{i}$ from $G_{3}$, the size of $\hat{V}_{3}$ (the set of vertices which are incident with at least $r / 30$ edges of $B_{1}, \ldots, B_{i}$ ) is small.

Again, let $S$ be a fixed vertex set of size $100 \ln n$ and $q_{k}$ be the probability that $S$ is visited by bundles $B_{1}, \ldots, B_{i}$ exactly $k$ times. We now evaluate the number of visits to $S$. Assume first that $\hat{a}_{i} \in S$. In this case this we will distinguish between free visits and start visits. $S$ undergoes $\ell$ visits, as the start point of all the walks in the bundle. All other visits to $S$ are free visits. For $k \geq 2$, the $k-\ell$ free visits to $S$ can be distributed among the $\ell$ walks in at most $\left(\begin{array}{c}k-1 \\ \ell-1\end{array}\right)$ ways. We have

$$
\operatorname{Pr}\left(k \text { visits and } \hat{a}_{i} \in S\right) \leq \frac{100 s}{n} \sum_{\ell=1}^{\infty}\left(\begin{array}{c}
k-1 \\
\ell-1
\end{array}\right) \rho_{S}^{k-\ell} \sigma(1-\sigma)^{\ell-1} \leq \frac{C_{4} s}{n}\left(1-\sigma+\rho_{S}\right)^{k-1}
$$


The term $\frac{100}{n}$ is arrived at as in (22).

Now consider the case $\hat{a}_{1} \notin S$. Here we distinguish between first visits and free visits. A first visit is the first visit to $S$ in a walk. Now we argue that there will be at least $k-\ell$ free visits and the probability of actually visiting $S$ at least once in $\ell$ walks it at most $\frac{1000 s \ell \log _{r} n}{n}$. We obtain

$\operatorname{Pr}\left(k\right.$ visits and $\left.\hat{a}_{i} \notin S\right) \leq \sum_{\ell=1}^{\infty} \frac{1000 s \ell \log _{r} n}{n}\left(\begin{array}{c}k-1 \\ \ell-1\end{array}\right) \rho_{S}^{k-\ell} \sigma(1-\sigma)^{\ell-1} \leq \frac{C_{5} s \log _{r} n}{n}\left(1-\sigma+\rho_{S}\right)^{k-1}$.

So putting $\xi=\left(\sigma-\rho_{S}\right) / 2>1 / 20$ we see that

$$
\begin{aligned}
\mathbf{E}\left((1+\xi)^{N_{i, S}}\right. & \leq 1+\sum_{k \geq 1} \frac{C_{6} s \log _{r} n}{n}(1-\xi)^{k-1} \\
& \leq 1+\frac{C_{6} s \log _{r} n}{n}
\end{aligned}
$$

Arguing as from (25) to (26) we then obtain that

$$
\operatorname{Pr}\left(\sum_{j=1}^{\kappa} Z_{j, S} \geq s t \mid \mathcal{E}^{(I)}, \overline{\mathcal{P}}\right) \leq 2(1+\xi)^{s\left(-t / 2+2 \alpha r C_{6}\right)} .
$$

We then take $t=r / 30$ as before and obtain

$$
\operatorname{Pr}\left(S \subseteq \hat{V}_{3} \mid \mathcal{E}^{(i)}, \overline{\mathcal{P}}\right) \leq 2(1+\xi)^{-r s / 120}<2 \epsilon_{2}
$$

We then argue, as before, that whp $\left|\hat{V}_{3}\right| \leq 3 \epsilon_{2} n$ and then use Lemma 3 to show that the $\lceil r / 10\rceil$-core of $G_{3}, G_{4}$ is of size at least $n-\epsilon_{K} n$.

\subsection{Analysis of CONNECTBACK}

We use the subroutine CONNECTBACK to connect vertices which are not in $K$ back to $K$. In this subroutine we construct random walks using only uncovered edges. This effectively removes the possibility of long walks which go back and forward over the same edges.

Recall the configuration model of Section 5.1. Assuming (10) and

$$
\left|V\left(\Gamma_{5}\right)\right| \geq n-n e^{-r / 800}-o(n)
$$

at each step of one of the random walks in CONNECTBACK, the probability that the next vertex of the walk is not in $K$ is at most $3 \epsilon_{K}$. The degrees of $\Gamma_{5}$ satisfy (7) and these walks model Construct. (31) is justified by Lemma 4 and the fact that whp the $\lceil r / 10\rceil$-core of $\Gamma_{5}$ contains all but $o(n)$ vertices of $G_{5}$ - see the argument preceding (33) below. The main algorithm GENPATHS will call the subroutine ConNECTBACK at most $4 \kappa$ times, so that the total number of random walks executed by CoNNECTBACK is at most $4 \kappa$. Let $W_{i}$ be the $i$ th walk executed by ConNectBACK. As before, fix $v$ and let $N_{i, v}$ be the number of visits to $v \in G_{5}$ by the walk $W_{i}, Z_{i, v}$ be the number of edges incident with $v$ which are traversed by $W_{i}$ and let $\ell_{i}$ be the length of $W_{i}$. It is clear from the previous paragraph that $\operatorname{Pr}\left(\ell_{i} \geq(\ln n)^{2}\right)=O\left(n^{-A}\right)$ for any constant $A>0$. We can therefore consider a modified algorithm which terminates in failure if ever we find $\ell_{i} \geq(\ln n)^{2}$. 
Note now that the probability that $W_{i}$ starts at $v$ is at most $10 / n$ since either $v$ is chosen uniformly from $K^{(0)}$ (Step 6 in GenPATHS) or it is the endpoint of a long random walk (Steps 15, 17). Then

$$
\begin{aligned}
\operatorname{Pr}\left(N_{i, v}=k\right) & =\sum_{\ell=k}^{(\ln n)^{2}} \operatorname{Pr}\left(N_{i, v}=k, \ell_{i}=\ell\right) \\
& \leq \sum_{\ell=k}^{(\ln n)^{2}}\left(3 \epsilon_{K}\right)^{\ell-1}\left(1-3 \epsilon_{K}\right)\left(\begin{array}{l}
\ell \\
k
\end{array}\right)\left(\frac{10}{n}\right)^{k} \\
& \leq \frac{\left(3 \epsilon_{K}\right)^{k-1}}{\left(1-3 \epsilon_{K}\right)^{k}}\left(\frac{10}{n}\right)^{k} \\
& \leq \frac{10}{n^{k}}
\end{aligned}
$$

In (32) we have $\left(3 \epsilon_{K}\right)^{\ell-1}\left(1-3 \epsilon_{K}\right)$ as a bound for the probability that $\ell_{i}=\ell$. Then there are $\left(\begin{array}{l}\ell \\ k\end{array}\right)$ positions on the walk for the visits to $v$. Then $10 / n$ bounds the probability that the walk makes the move to $v$ at this point.

Note that if $v \in K$ then $N_{i, v} \leq 1$. Also, the upper bound we have calculated is valid, conditional on the previous history of the algorithm.

Suppose that there are $\kappa_{1} \leq 4 \kappa$ walks generated by ConNECTBACK overall. Then, for any $t>0$

$$
\begin{aligned}
\operatorname{Pr}\left(\sum_{j=1}^{\kappa_{1}} N_{j, v}=t\right) & \leq \sum_{\ell_{1}+\cdots+\ell_{\kappa_{1}}=t} \prod_{j=1}^{\kappa_{1}}\left(\frac{10}{n}\right)^{\ell_{j}} \\
& \leq\left(\begin{array}{c}
\kappa+t-1 \\
t
\end{array}\right)\left(\frac{10}{n}\right)^{t} \\
& \leq\left(\begin{array}{c}
4 \kappa+t-1 \\
t
\end{array}\right)\left(\frac{10}{n}\right)^{t} \\
& \leq\left(\frac{40 e \alpha r}{\log _{r} n}\right)^{t}
\end{aligned}
$$

Let $\hat{V}_{5}$ be the set of vertices of $G_{5}$ which are incident with at least $r / 30$ edges of $W_{1}, \ldots, W_{i}$.

Then

$$
\begin{aligned}
\operatorname{Pr}\left(v \in \hat{V}_{5}\right) & \leq \operatorname{Pr}\left(\sum_{j=1}^{\kappa} N_{j, v} \geq r / 30\right) \\
& \leq \sum_{t=r / 30}^{2 r / 7} \operatorname{Pr}\left(\sum_{j=1}^{\kappa} N_{j, v}=t\right) \leq 2\left(\frac{40 e \alpha r}{\log _{r} n}\right)^{r / 30}
\end{aligned}
$$

So if $\rho=n\left(\frac{40 e \alpha r}{\log _{r} n}\right)^{r / 40}$ then, by the Markov inequality,

$$
\operatorname{Pr}\left(\left|\hat{V}_{5}\right| \geq \rho\right) \leq\left(\frac{40 e \alpha r}{\log _{r} n}\right)^{r / 120} \rightarrow 0
$$

If $\hat{V}_{5}$ is this small then Lemma 3 implies that the $\lceil r / 10\rceil$-core $\Gamma_{5}$ contains all but $2 \rho$ vertices of $G_{5}$ (see the argument following (27)). 
Thus

$$
|L| \leq 2 \rho \mathbf{w h p}
$$

as $L \subseteq G_{5} \backslash \Gamma_{5}$.

\section{Analysis of Phase 4}

We join the pairs in $L$ using the algorithm of [10]. The algorithm is capable of joining $\Omega\left(n /(\ln n)^{c}\right)$ distinct pairs for some constant $c>0$, provided the graph has sufficent edgeexpansion. Notice that $\tilde{a}_{i}, \tilde{b}_{i}$ are chosen as distinct vertices. For large $r, G_{6}$ will $\mathbf{w h p}$ have ample expansion and $2 \rho$ will be small enough for the algorithm.

\section{References}

[1] N. Alon, J. Spencer and P. Erdős, The probabilistic method, John Wiley and Sons, 1992.

[2] E. A. Bender and E. R. Canfield, The asymptotic number of labelled graphs with given degree sequences, Journal of Combinatorial Theory (A) 24, (1978) 296-307.

[3] B. Bollobás, Random graphs, Academic press, 1985.

[4] B. Bollobás, A probabilistic proof of an asymptotic formula for the number of labelled regular graphs, European Journal on Combinatorics 1, (1980) 311-316.

[5] B. Bollobás, Martingale, isoperimetric inequalities and random graphs, Coll. Math. Soc. J. Bolyai 52, 113-139 (1987).

[6] B. Bollobás, T. I. Fenner, and A. M. Frieze, Hamilton cycles in random graphs with minimal degree at least $k$, in "A tribute to Paul Erdős," edited by A. Baker, B. Bollobás, and A. Hajnal (1990) 59-96.

[7] J. A. Bondy and U. S. R. Murty, Graph Theory with Applications, North-Holland 1976 .

[8] A. Z. Broder, A. M. Frieze, S. Suen, and E. Upfal, Optimal Construction of EdgeDisjoint Paths in Random Graphs, to appear.

[9] A. Z. Broder, A. M. Frieze, S. Suen, and E. Upfal, Optimal construction of vertexdisjoint paths in random graphs, in preparation.

[10] A. Z. Broder, A. M. Frieze, and E. Upfal, Existence and construction of edge disjoint paths on expander graphs, SIAM Journal on Computing 23 (1994) 976-989.

[11] A. Z. Broder, A. M. Frieze, and E. Upfal, Static and dynamic path selection on expander graphs: a random walk approach, to appear.

[12] P. Erdős and L. Lovász, Problems and results on 3-chromatic hypergraphs and some related questions. In A. Hajnal et al., editors, Infinite and Finite Sets, volume 11 of Colloq. Math. Soc. J. Bolyai, pages 609-627. North Holland, 1975. 
This research was sponsored in part by National Science Foundation (NSF) grant no. CCR-0122581. 